\title{
Detection of Thermal Responses of the Retina by Use of Polyvinylidene Fluoride Multilayer Detector
}

\author{
Ichiji Tasaki, Paul M. Byrne, and Michio Masumura \\ National Institute of Mental Health, Bethesda, \\ Maryland, 20892 U.S.A.
}

\begin{abstract}
The design and properties of thermal detectors constructed with multiple layers of polyvinylidene fluoride film are described. By use of these detectors, production of heat by the photoreceptors in the darkadapted bullfrog retina in response to brief pulses of very weak light was examined. In response to brief light pulses delivering an average of 1 photon per retinal rod, the retina was found to produce heat more-or-less abruptly after a latent period of 0.2 to $0.4 \mathrm{~s}$. At this level of pulse intensity, the thermal energy produced by the retina was approximately $1,500,000$ times as large as the total radiant energy delivered to the retina for stimulation. It appears possible that a large number of disks in the rod outer segment are activated by absorption of a single photon.
\end{abstract}

Key words: heat detector, retinal rod, thermal response.

Polyvinylidene fluoride (PVDF) is a synthetic polymeric material which becomes highly piezo- and pyroelectric when heated and stretched in the presence of a high electric field. This material has been used for detection of brief pulses of infrared radiation (GLASS et al., 1971; PETERSON et al., 1974). Using heat sensors constructed with PVDF film, we found it possible to detect production of heat by various excitable tissues during their physiological activities (TASAKI and IWASA, 1981; TASAKI and NAKAYE, 1985). With a simple type of PVDF thermal detector, we could record thermal responses of the dark-adapted bullfrog retina to relatively strong light pulses (TASAKI and NAKAYE, 1986).

Quite recently, we have constructed PVDF thermal detectors with a multilayer configuration (TASAKI and BYRNE, 1987). By using these improved detectors, we could extend our thermal measurements on retinae to light intensities far lower than those employed in previous studies. In the present paper, we first describe the design and properties of these multilayer thermal detectors. Next, we discuss properties of the thermal responses of the dark-adapted bullfrog retina to weak light pulses, as revealed by use of these detectors. Finally, we discuss the possile relationship

Received for publication March 30, 1987 
between the process of heat production by the rods and the development of electric responses of the retina.

\section{MATERIALS AND METHODS}

(1) Design and properties of multilayer PVDF thermal detectors. The 9- $\mu \mathrm{m}$ thick PVDF film used in the present study was manufactured by Kureha Chemical Industries, Horidome-cho, Tokyo. The film was already stretched and poled, and has an approximately 10 -nm thick aluminum layer deposited on each surface. According to the technical data supplied by the manufacturer, this polymeric material has the following physical properties: density $1.78-1.79 \mathrm{~g} / \mathrm{cm}^{3}$, dielectric constant $12-13$, volume resistivity roughly $9 \times 10^{14} \Omega \cdot \mathrm{cm}$, heat capacity $0.57 \mathrm{cal} \cdot \mathrm{cm}^{-3} \cdot \mathrm{deg}^{-1}$, thermal diffusivity $0.53 \times 10^{-3} \mathrm{~cm}^{2} / \mathrm{s}$. The pyroelectric coefficient, i.e. the change in electric charge on the surface of the film induced by one degree change in the ambient temperature, is 4 to 5 times $10^{-9} \mathrm{C} /\left(\mathrm{deg} \cdot \mathrm{cm}^{2}\right)$. At present, PVDF film with comparable quality can be purchased from Pennwalt Corp. under the commercial name of "Kynar."

The thermal detectors employed in the present study were constructed by using four or eight layers of PVDF film. The procedure for making a four-layer detector is as follows. From a large sheet of PVDF film, a $16 \times 16 \mathrm{~mm}^{2}$ piece with a $3 \times 8 \mathrm{~mm}^{2}$ appendage (see Fig. 1A) was cut. A very thin layer of " 5 min Epoxy" (Devcon) was applied to one of the surfaces of the rectangular portion of the PVDF sheet; immediately, the sheet was folded and a mild pressure was applied to the sheet until the two surfaces were glued together. Next, on one surface of the folded PVDF sheet, a fresh layer of Epoxy resin was applied and the sheet was folded and glued again. Finally, a rectangular piece of silver plate, $10 \mu \mathrm{m}$ in thickness and $8 \times 8 \mathrm{~mm}^{2}$ in size, was glued on one surface fo the folded PVDF sheet. The entire thickness of this multilayer structure was roughly $50 \mu \mathrm{m}$. It was important to ascertain at this stage that the folding procedure did not break the aluminum layers on the surface of the PVDF film; this was done by demonstrating that the electric capacity of the film remained unaltered during the procedure. Note that the well-known formula for a parallel plate capacitor can be written in the present case as $C=s /(0.9 \times d)$, where $C$

A

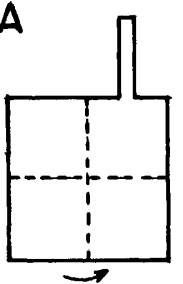

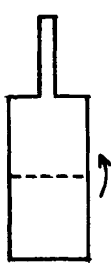

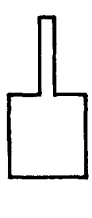

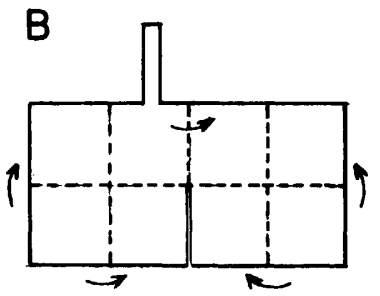

Fig. 1. Diagram illustrating the procedure for folding PVDF film in fabrication of four-layer (A) and eight-layer (B) heat-sensors. See the text for details. 
is the capacity $(\mathrm{pF}), s$ is the area $\left(\mathrm{cm}^{2}\right)$, and $d$ the thickness $(\mathrm{cm})$ of the PVDF film, for the dielectric constant of PVDF is numerically close to $4 \pi$. The capacity of this heat sensor was approximately $3.6 \mathrm{nF}$.

The procedure of fabricating an eight-layer heat sensor was very similar to that described above. We used a large piece of PVDF film $\left(16 \times 32 \mathrm{~mm}^{2}\right.$ plus appendage $)$ with a partial slit in the middle (see Fig. 1B). This slit was made in order to avoid an excessive tension developed by the film when the folding procedure was completed. The capacity of this film was about $7.5 \mathrm{nF}$.

A large sheet of $7 \mu \mathrm{m}$ thick Mylar was adhered to one surface of a black Lucite plate with a large (approximately $15 \mathrm{~mm}$ in diameter) hole in the middle (see Fig. 2, right). The Mylar sheet used was an insulating layer found inside a commercially available capacitor and had an approximately 10-nm-thick aluminum layer deposited on one surface. The sheet was attached to the Lucite plate with its aluminum-deposited surface facing downwards. It was important that the Mylar sheet covering the hole was taut and free of wrinkles; the use of a layer of a 3M \#666 double coated tape (with adhesive surfaces on both sides) between the Lucite plate and the Mylar sheet yielded the desired result.

Next, the silver plate on the surface of the folded PVDF sheet was glued to the aluminum-deposited surface of the Mylar sheet. The objective of inserting a rigid metal plate between the Mylar sheet and the PVDF film was to ensure a uniform rise of the temperature of the film and to reduce mechanical disturbances arriving at the film. In most cases, the upper surface of the Mylar sheet was sprayed with "Uralane 8267 Circuit Coate" (Furane Product Co.) for protection against abrasion. A thick
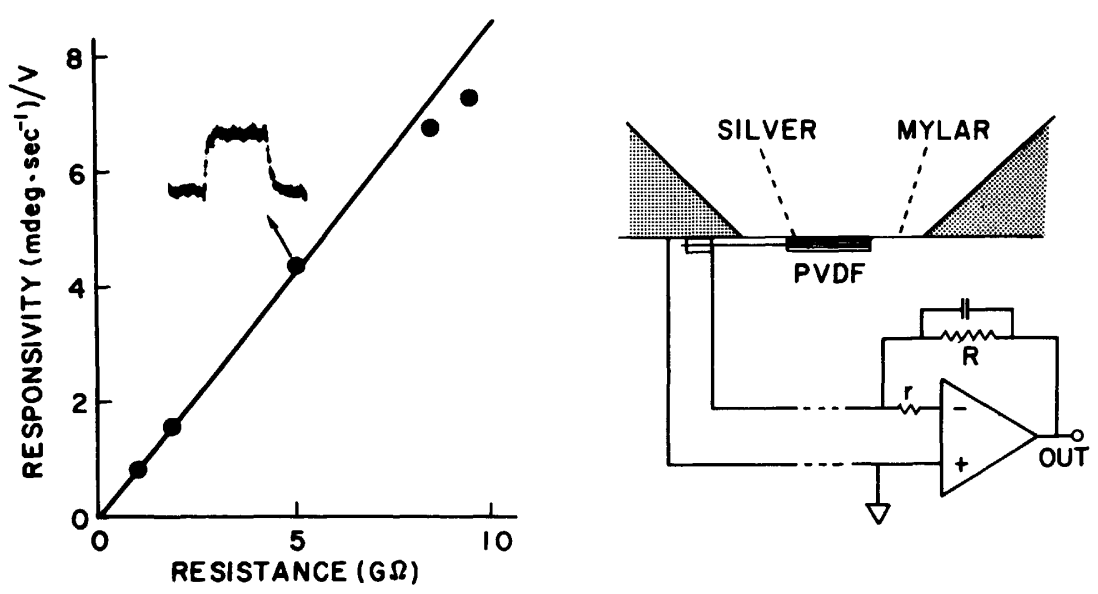

Fig. 2. Schematic diagram showing the arrangement employed for detection of heat production by the retina (right) and data demonstrating the dependence of the responsivity of a four-layer PVDF thermal detector on the feed-back resistance (left). The resistance used for input protection ( $r$ in the figure) was $80 \mathrm{k} \Omega$. A record of response of the detector to a pulse of Joule's heat ( $1 \mathrm{~s}$ in duration) is shown. The time constant (RC-product) of the amplifier was kept at $10 \mathrm{~ms}$ throughout. See the text for details. 
layer of foam rubber was used to press the assembly of PVDF film from below (not shown in the figure).

The heat sensor constructed with multiple layers of PVDF film was connected to an operational amplifier as illustrated in Fig. 2, right. We employed a narrow strip of thin copper sheet (in conjunction with a small plastic clamp) to connect the appendage of the PVDF sheet to the amplifier input. The operational amplifier employed was either a OPA111 (Burr-Brown) or AD515 (Analog Devices). Note that the silver plate, as well as the aluminum layer on the Mylar sheet, was connected to the ground terminal of the amplifier. The feedback resistance ( $R$ in the figure) of the amplifier was between 1 and $10 \mathrm{G} \Omega$. In parallel with the feedback resistor, a small capacitor ( 1 to $10 \mathrm{pF}$ depending on the time resolution required) was connected. To reduce current leakage, the feedback resistor was mounted on Teflon insulated terminals. It was also important to electrically shield the amplifier circuit. The output of the amplifier was led to a single-average via a D.C.-coupled amplifier with a gain of 10 or an A.C.-amplifier with a gain of 100. Precautions were taken to reduce acoustic disturbances arriving at the amplifier input.

The output voltage of the thermal detector described above is proportional to the rate of change of the temperature of the PVDF probe (RoUNDY, 1975; TASAKI and NAKAYE, 1986). The responsivity of the detector was determined by converting the radiant energy of a light pulse into heat in the heat-sensitive area of the detector. For this purpose, we employed a uniform layer of aqueous solution containing a mixture of phenol red and chlorphenol red (about $50 \mathrm{mg}$ each.dissolved in $100 \mathrm{ml}$ of $\mathrm{pH}$ buffer at $\mathrm{pH}$ 7.4) covering the heat-sensitive area of the detector in conjunction with pulses of $500 \mathrm{~nm}$ light (which was completely absorbed by the dye solution). A thermal scale of the observed deflection $(\mathrm{deg} / \mathrm{s})$ was obtained by dividing the radiant energy arriving at the detector surface (W) by the heat capacity of the dye solution (approximately $4.2 \mathrm{~J} \cdot \mathrm{deg}^{-1} \cdot \mathrm{cm}^{-3}$ times the volume of the dye solution). The heat generated by the retina was calibrated by this method.

Alternatively, the device can be calibrated by using production of Joule's heat in a thin layer of electrolyte solution carrying a pulse of electric current (TASAKI and IWASA, 1981). We employed this method to examine the dependence of the responsivity of the detector on its feedback resistance (Fig. 2, left). A uniform layer of $2 \%$ agar-Ringer gel (about $0.5 \mathrm{~mm}$ thick and $8 \mathrm{~mm}$ wide) traversed by 1 -s-long current pulses (about $0.7 \mathrm{~mA}$ ) was used in this case. Under these conditions, the output voltage of the detector was proportional to the square of the current intensity (irrespective of the direction of the current). It can be seen in the figure that, within a wide range, the responsivity is proportional to the feedback resistance. A small deviation from the proportionality is observed when very high feedback resistances were used. This deviation is due evidently to current leakage.

The responsivity of an eight-layer thermal detector is roughly twice as high as that of a four-layer detector. However, it is to be noted that this enhancement of the responsivity is accompanied by an increase in the response time of the detector. Note that $x^{2} /(2 D)$, where $D$ is the thermal diffusivity, can be used as a measure of 
time required for conduction of heat over a distance of $x \mathrm{~cm}$ (see TASAKI and NAKAYE, 1986). In a detector with eight layers of PVDF film and a layer of Mylar sheet, this measure of time required for the heat to raise the temperature of the lowermost PVDF layer is about $80 \mathrm{~ms}$. Note that the thermal diffusivity of silver is three orders of magnitude greater than that of the polymers; hence the silver layer does not affect the response time. Evidently, the temperature of a PVDF sheet near the top rises more rapidly. The overall thermal response time of the eight-layer detector is considered to be about $25 \mathrm{~ms}$.

The random noise at the output of the detector is expected to be governed by the Johnson noise of the feedback resistor and/or the voltage noise of the amplifier (Roundy, 1975). Frequently, however, the amplifier output is dominated by much larger oscillatory potential variations which are probably caused by a small mechanical vibration of some part(s) of the detector. It was necessary to make the cable systems vibration-free and to reduce mechanical disturbances arriving at the detector.

(2) Method of recording thermal responses of the retina. Dark-adapted retinae of the bullfrog, Rana catesbeiana, were isolated in oxygenated Ringer's solution under a dim red light. The composition of the Ringer most frequently used contained (in mM) $110 \mathrm{NaCl}, 1.5 \mathrm{KCl}, 1.0 \mathrm{CaCl}_{2}, 5$ glucose, and $10 \mathrm{HEPES}$ (at pH 7.3-7.4). In order to block the synapses at the proximal ends of the photoreceptor cells, the retina was immersed in a 1:1 mixture of an isotonic $\mathrm{MgCl}_{2}$ and normal Ringer's solutions (PINTO and PAK, 1974; TASAKI and NAKAYE, 1986) for about 6 min before it was introduced into a thermal detector. The outer (receptor) surface of the retina was brought in direct contact with the heat-sensitive area of the detector. The Ringer around the retina was removed with blotting paper. A gentle flow of moist oxygen was maintained above the retina.

Pulses of light for stimulation were obtained from an Osram quartz-iodine lamp $(100 \mathrm{~W})$ used in conjunction with an electromechanical shutter. The time required for opening or closing of the shutter was about $3 \mathrm{~ms}$. The lamp was operated with storage batteries $(12 \mathrm{~V})$. The wavelength of the light was selected by inserting an interference filter in the pathway of the light. The light intensity was varied both by varying the voltage operating the lamp and by using neutral density filters. The light was led to the retina with a long light guide. The light intensity at the level of the retina was calibrated by using a Model 550 photometer (E. G. \& G. Electro-Optics). The average number of photons arriving at individual retinal rods was estimated by taking the mean cross-sectional area of the rod to be $3.8 \times 10^{-7} \mathrm{~cm}^{2}$ (see BowNDS and BRODIE, 1975). When a four-layer thermal detector was used, thermal responses were recorded after signal-averaging over 2-8 trials repeated at intervals of about $14 \mathrm{~s}$ (Figs. 3 and 5). When an eight-layer detector was employed (Fig. 4), records of thermal responses were taken without averaging. All the experiments were carried out at room temperature $\left(21-23^{\circ} \mathrm{C}\right)$. 


\section{RESULTS}

Figure 3 shows an example of thermal responses of $\mathrm{Mg}^{2+}$-treated retinae to brief pulses of quasi-monochromatic light of moderate and low intensities. These records were taken by using a four-layer PVDF heat-sensor connected to an operational amplifier with a time constant of $10 \mathrm{~ms}$. The optical densities of the neutral density filters used, as well as the unattenuated intensity of light, are given in the figure. The two upper traces in the records represent the rate of temperature rise which reflects the time-course of heat production by the retina. The lower traces, obtained by integration of the upper traces, represent the change in temperature at the outer retinal surface (which is in contact with the heat-sensor) following delivery of the light pulse.

It is seen in the figure that, when the light pulse is relatively strong, thermal responses (represented by deflections of the upper traces) start shortly after the onset of the light stimulus. As has been noted previously (TASAKI and NAKAYE, 1986), thermal responses observed under these conditions consist of two phases: the initial rapid deflection of the trace (fast phase) is followed by a slow return of the trace to the baseline (slow phase). In the top trace in Fig. 3, left, the fast phase is seen to terminate at about $300 \mathrm{~ms}$ after delivery of the light pulse.

When the intensity of the stimulating light pulse was reduced step-by-step, the transition from the fast phase of the response to the slow phase was found to become gradually obscure. With very weak light pulses, only slow thermal responses were observed (see the right-hand record in Fig. 3). It is to be noted that, even at low

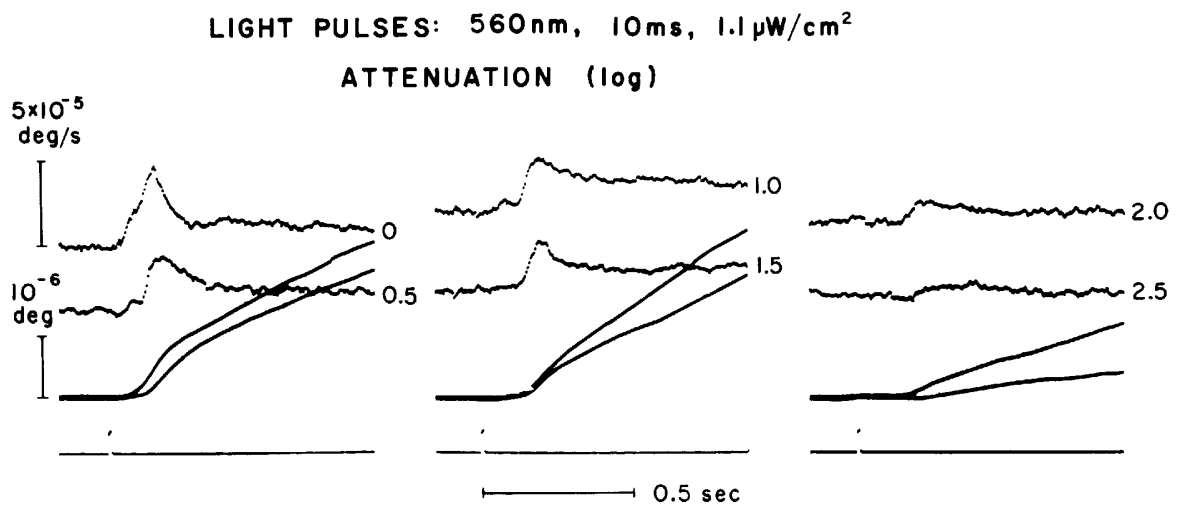

Fig. 3. Thermal responses of a dark-adapted bullfrog retina (treated with a $1: 1$ mixture of isotonic magnesium chloride solution and Ringer) to brief light pulses. The wavelength of the light, the pulse duration, and the unattenuated light intensity employed are given. The two traces on the top of each record show the rate of temperature rise on the outer surface of the retina. The two lower (smooth) traces, obtained by integration of the upper traces, indicate the changes in the temperature following delivery of light pulses (marked by the bottom traces). Each trace in the records represents an average of four responses. 
LIGHT PULSES: $500 \mathrm{~nm}, 10 \mathrm{msec}, 0.22 \mu \mathrm{W} / \mathrm{cm}^{2}$

ATTENUATION (log)
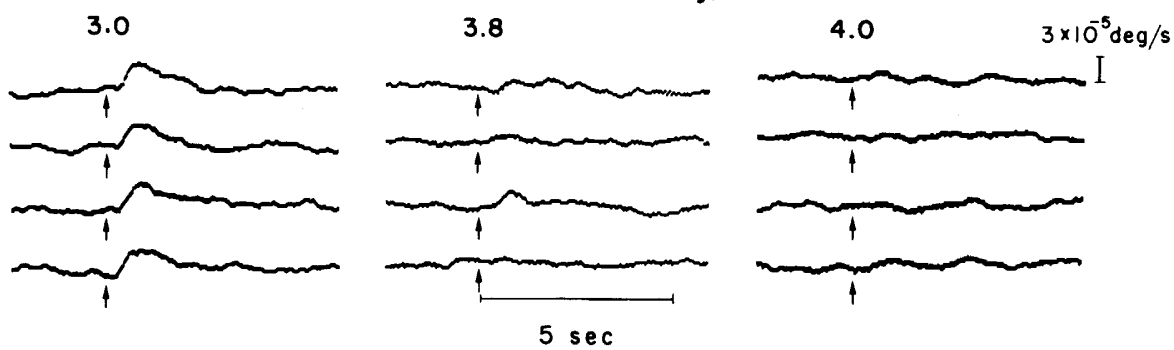

Fig. 4. Single-sweep records of thermal responses of a dark-adapted bullfrog retina treated with a high magnesium salt solution to weak light pulses. The four traces in each column were taken at about 40-s intervals using light pulses of the same intensity. The thermal responses shown in the left column were triggered by one photon per rod on an average. An eight-layer PVDF thermal detector with a time constant of $50 \mathrm{~ms}$ was used for recording.

levels of stimulus intensity, the onset of a thermal response was fairly abrupt.

It is of interest to investigate properties of the thermal responses evoked by very weak light pulses that are expected to deliver one photon per rod on an average. Figure 4 shows an example of the records obtained by using light pulses with such low intensities. In each column in the figure, there are four single-sweep records of thermal responses taken under the identical experimental conditions. The intensity of the 500-nm light pulse with which the records in the left-hand column were taken was $2.2 \times 10^{-10} \mathrm{~W} / \mathrm{cm}^{2}$ at the position of the retina. This light pulse $(10 \mathrm{~ms}$ in duration) delivers an average of 2 photons to a rod with a diameter of $7 \mu \mathrm{m}$. If a single absorbed photon triggers a response with an efficiency of 0.5 (BAYLOR et al., 1979), the majority of the rods are expected to be excited by only one photon.

A large number of thermal responses were recorded from many dark-adapted retinae under these conditions. The latent period of the responses observed was found to vary between 0.2 and $0.4 \mathrm{~s}$ among different preparations. The rate of temperature rise at the peak of these responses was usually between 1.1 and 2.0 times $10^{-5} \mathrm{deg} / \mathrm{s}$. These responses were found to last for 0.7 to $1.5 \mathrm{~s}$.

When the intensity of the light pulse was decreased below the above-cited level, both the magnitude and the time-course of the response became variable. The records in the middle column in Fig. 4 were taken by using light pulses that are expected to deliver $1 / 3$ photon per rod on an average. The retina responded only occasionally under these conditions. When the light intensity was decreased further, it became difficult to record thermal responses.

The dependence of the peak value of the thermal response on the light intensity was examined using $500-\mathrm{nm}$ light pulses of $10 \mathrm{~ms}$ in duration. In the range of intensity between 100 and about 2 absorbed photons per rod, the peak value was found to fall, in most cases, by a factor of 1.7 to 2.2 when the intensity was reduced 
LIGHT PULSES: $\quad 500 \mathrm{~nm}, 10 \mathrm{msec}$

$2.2 \times 10^{-10} \mathrm{~W} / \mathrm{cm}^{2}$

$4.6 \times 10^{-5} \mathrm{~W} / \mathrm{cm}^{2}$

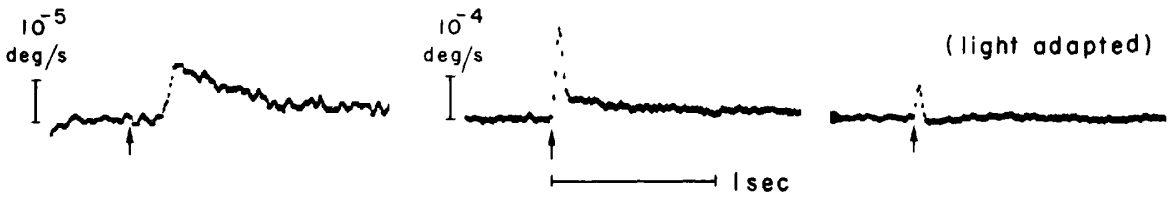

Fig. 5. Thermal responses of a dark-adapted bullfrog retina treated with a high magnesium salt solution to weak (left) and strong (middle) light pulses. The wavelenghth, the duration and the intensities of the light pulses employed are given. The right-hand record was taken after 5-min exposure of the retina to room light: the heat signal observed under these conditions is caused predominantly by direct conversion of the radiant energy of the stimulating light pulse into heat. By subtraction of this "direct heat" from the signal in the middle, the peak amplitude of the large response is found to be about $15 \%$ smaller than the peak of the large deflection in the middle.

to $1 / 10$. This value is not significantly different from that obtained previously in high and intermediate ranges of the light intensity (TASAKI and NAKAYE, 1986).

Figure 5 shows a thermal response evoked by a very strong light pulse in comparison with the smallest (stable) response described above. A four-layer heatsensor connected to an operational amplifier with a time constant of $10 \mathrm{~ms}$ was used for recording. The intensity of the strong light pulse employed was five orders of magnitude greater than that of the weak pulse. Note the difference in the timecourse, as well as in the magnitude, between the two responses in the figure. The large deflection shown in the middle of the figure contains a component of heat generated by direct conversion of the radiant energy absorbed predominantly by the photopigments (TASAKI and NAKAYE, 1986); the "direct heat" is the major component in the deflection taken after light-adaptation of the retina. It is seen that the peak value of the large response was approximately 18 times as large as that of the small response. The duration of the fast phase of the large response was roughly $0.1 \mathrm{~s}$; its total duration was longer than $10 \mathrm{~s}$.

\section{DISCUSSION}

The multilayer heat-sensor described under MATERIALS AND METHODS have a high sensitivity and a reasonably short response time. By using these heatsensors, properties of thermal responses of $\mathrm{Mg}^{2+}$-treated retinae to brief pulses of very weak light were examined. An inevitable question comes to mind-what is the relationship between these thermal responses and the electrical responses of the retina?

In the previous paper (TASAKI and NAKAYE, 1986), we have described several pieces of evidence indicating that the thermal response is a sign of an exothermic 
process which precedes the generation of electrochemical changes in the plasma membrane of the rods. Removal of $\mathrm{Na}$-ion or raising the $\mathrm{K}$-ion concentration in the external medium, which suppresses electrical responses, does not eliminate thermal responses. The peak of the electrical response lags clearly behind the peak of the thermal response. The wavelength of the light most effective in eliciting a thermal response is approximately $500 \mathrm{~nm}$, which coincides with the absorption maximum of the red rod (Harosi, 1975). From these and other findings, we presume that the thermal response is triggered by absorption of photons by rhodopsin molecules in the red rod. The product ensuing from the exothermic process leads to changes in the electrochemical properties of the plasma membrane.

It is generally held that the generation of an electrical response proceeds with a great amplification of energy involved (Hagins et al., 1970; PugH and CoBBs, 1986). At the step of generation of a thermal response, we have shown previously that there is an enormous amplification of energy. In the present study, we have determined the total amount of heat evolved in association with the smallest (stable) response by a fairly direct means. We made a small pool of dye solution covering the heat-sensitive area of the detector and applied a pulse of light that is strongly absorbed by the dye (see MATERIALS AND METHODS). When the heat capacity of the dye solution is nearly the same as that of the retina under study, the energy of the light pulse required to produce the same temperature rise as that observed at the end of a thermal response gives a direct measure of the energy generated by the retina.

We have found by this procedure that the total amount of heat generated in association with the smallest thermal response of a $8 \times 8 \mathrm{~mm}^{2}$ piece of retina was approximately $5 \times 10^{-7} \mathrm{cal}$. The radiant energy of the stimulus delivered to the retina under these conditions was $3.3 \times 10^{-13}$ cal. Thus, we find that the energy generated by the photoreceptors in the form of heat was approximately 1,500,000 times as large as the radiant energy used for stimulation. The average number of photons that was delivered to trigger this response was close to one per rod.

The mechanism by which a single absorbed photon triggers an exothermic process is an interesting puzzle for future investigation. The long latency and the more-or-less abrupt onset of the thermal response are reminiscent of the process of initiating an electrical response in axonal membranes by use of a barely suprathreshold electric pulse. In the axonal membrane, the occurrence of a phasetransition (first order) has been postulated (TASAKI, 1982). It is tempting, consequently, to suggest a similar mechanism for the process of triggering a thermal response.

There are a number of similarities between intracellularly recorded electrical responses of the rod and the thermal responses. At high and intermediate stimulus intensities, electrical responses have a fast phase followed by a slow phase (see e.g. Cervetto et al., 1977) as thermal responses do. At very low light intensities, both responses are devoid of the fast phase. Both the latency and the duration of the two 
types of responses are comparable when the intensity of the stimulating light pulse is very low.

It is widely accepted that disks in the rod outer segment are separated from the neighboring disks, as well as from the plasma membrane, by narrow cytoplasmic space (see e.g. OLIVE, 1980). However, if the exothermic process commenced in one disk is assumed to be localized to the space occupied by the disk, it seems impossible to explain why the thermal response evoked by a very weak light pulse is so large that its peak amplitude is nearly a few percent of a few percent of the largest response (see Fig. 5). If the exothermic process in one disk does not spread to the neighboring disks, the response amplitude is expected to double when the intensity of a very weak stimulus is doubled. For this reason, we hypothesize that there is a mechanism by which the exothermic process initiated in one disk spreads to the next. The disk membrane may be activated by two distinct means, directly by photon absorption and indirectly by the spread of the exothermic process from neighboring disks. The existence of two phases in the thermal response may be interpreted on the basis of this hypothesis, for direct activation by a great number of photons is likely to evoke an exothermic process that proceeds faster than an indirectly induced process.

The nature of the process of triggering a thermal response without involving photon absorption is not clear at present. In the sarcoplasmic reticulum of the muscle, it is known that calcium release is a regenerative process in which calcium itself causes the release of calcium from the reticulum (ENDo et al., 1970). The release of calcium by the disk (GOLD and KorENBROT, 1980; YoshiKAMI et al., 1980) combined with the proposal of calcium-induced calcium-release appears to offer a possible explanation of the process. Further studies are required to explain the development of the exothermic process in terms of the postulate of increased guanine metabolic flux (GolDBERG et al., 1983) or on the basis of the cyclic nucleotide cascade mechanism (STRYER et al., 1981).

We thank Mr. T. Araki of Kureha Chemical Ind. Co. for the gift of PVDF film.

\section{REFERENCES}

BaYloR, D. A., LAmb, T. D., and YAU, K.-W. (1979) Responses of retinal rods to single photons. J. Physiol. (Lond.), 288: 613-634.

Bownds, D. and BRoDie, A. E. (1975) Light-sensitive swelling of isolated frog rod outer segments as an in vitro assay for visual transduction and dark adaptation. J. Gen. Physiol., 66: 407-425.

Cervetto, L., Pasino, E., and Torre, V. (1977) Electrical responses of rods in the retina of Bufo marinus. J. Physiol. (Lond.), 267: 17-51.

Endo, M., Tanaka, M., and Ogawa, Y. (1970) Calcium induced release of calcium from the sarcoplasmic reticulum of skinned skeletal muscle fibres. Nature, 228: 34-36.

Glass, A. M., McFee, J. H., and Bergman, J. G., Jr. (1971) Pyroelectric properties of polyvinylidene fluoride and its use for infrared detection. J. Appl. Phys., 42: 52195222. 
Gold, G. H. and Korenbrot, J. I. (1980) Light-induced calcium release by intact retinal rods. Proc. Natl. Acad. Sci. U.S.A., 77: 5557-5561.

Goldberg, N. D., Ames, III, A., Gander, J. E., and Walseth, T. F. (1983) Magnitude of increase in retinal cGMP metabolic flux determined by ${ }^{18} \mathrm{O}$ incorporation into nucleotide $\alpha$-phosphoryls corresponds with intensity of photic stimulation. J. Biol. Chem., 258: 9213-9219.

Hagins, W. A., Penn, R. D., YoshiKami, S. (1970) Dark current and photocurrent in retinal rods. Biophys. J., 10: $380-412$.

HaRosi, F. I. (1975) Absorption spectra and linear dichroism of some amphibian photoreceptors. J. Gen. Physiol., 66: 357-382.

OLIVE, J. (1980) The structural organization of mammalian retinal disc membrane. Int. Rev. Cytol., 64: 107-169.

Peterson, R. L., Day, G. W., Gruzensky, P. M., and Phelan, R. J., Jr., (1974) Analysis of response of pyroelectric optical detectors. J. Appl. Phys., 45: 3296-3303.

PINTO, L. H. and PAK, W. L. (1974) Light-induced changes in photoreceptor membrane resistance and potential in Gecko retinas. I. Preparations treated to reduce lateral interactions. J. Gen. Physiol., 64: 26-48.

Pugh, E. N., Jr. and CoBBs, W. H. (1986) Visual transduction in vertebrate rods and cones. A tale of two transmitters, calcium and cyclic GMP. Vision Res., 26: 1613-1643.

Roundy, C. B. (1975) Performance and uses of high speed pyroelectric detectors. Proc. Soc. Photo-Opt. Instrum. Eng., 62: 191-198.

Str yer, L., Hurley, J. B., and Fung, B. K.-K. (1981) First stage of amplification in the cyclic-nucleotide cascade of vision. In: Current Topics in Membranes and Transport, Vol. 15, pp. 93-108.

TASAKI, I. (1982) Physiology and Electrochemistry of Nerve Fibers, Academic Press, New York, pp. 348.

TASAKI, I. and BYRNE, P. M. (1987) Heat production associated with synaptic transmission in the bullfrog spinal cord. Brain Res., 407: 386-389.

TASAKI, I. and IWASA, K. (1981) Temperature changes associated with nerve excitation. Biochem. Biophys. Res. Commun., 101: 172-176.

TASAKI, I. and NAKAYE, T. (1985) Heat generated by the dark-adapted squid retina in response to light pulses. Science (Wash., D. C.), 227: 654-655.

TASAKI, I. and NAKAYE, T. (1986) Heat produced by the dark-adapted bullfrog retina in response to light pulses. Biophys. J., 50: 285-293.

Yoshikami, S., George, J. S., and Hagins, W. A. (1980) Light-induced calcium fluxes from outer segment layer of vertebrate retina. Nature (Lond.), 286: 395-398. 